\title{
A standardized formula for aesthetic mandibular reconstruction using an osteocu- taneous fibular free flap
}

\author{
Soung Min Kim ${ }^{1,2}$, Emmanuel K. Amponsah ${ }^{1}$, Hui Young Kim ${ }^{2}$, Ik Jae Kwon ${ }^{2}$, Hoon Myoung ${ }^{2}$ and \\ Jong Ho Lee ${ }^{2}$
}

Ghana Med J 2016; 50(3): 197-199 DOI: http://dx.doi.org/10.4314/gmj.v50i3.12

\begin{abstract}
${ }^{1}$ Oral and Maxillofacial Microvascular Reconstruction LAB, Brong Ahafo Regional Hospital, Sunyani, Ghana, ${ }^{2}$ Department of Oral and Maxillofacial Surgery, Dental Research Institute, School of Dentistry, Seoul National University, Seoul, Korea
\end{abstract}

Corresponding author: Dr. Jong Ho LEE

E-mail: leejongh@snu.ac.kr

Conflict of interest:

\section{SUMMARY}

Ameloblastoma is the most common benign odontogenic tumor of the jaw, and expansional growth of a huge untreated ameloblastoma can result in disturbances in facial aesthetics and function, such as difficulty with mouth opening, swallowing, chewing, breathing, neurologic deficits, and pathologic fractures. Radical wide resection with safety margins and subsequent reconstruction is generally recommended. A fibular free flap (FFF) is commonly used to reconstruct the mandible in order to adequately restore both aesthetic appearance and function. The aim of this brief clinical report is to present a case of huge ameloblastoma after wide resection with free safety margins, and describe the immediate one-step mandibular reconstruction using a vascularized composite FFF. The sterolithographic(rapid prototype, RP) model, a wax pattern of the resected mandible, and a surgical fibular stent made from the wax pattern were constructed preoperatively. We suggest a standardized surgical protocol for mandibular reconstruction with FFF.

Funding: Supported by the International Research \& Development Program of the National Research Foundation of Korea (NRF) funded by the Ministry of Science, ICT \& Future Planning (NRF-2015K1A3A9A01028230)

Keywords: Mandibular reconstruction, fibular osteocutaneous free flap, huge ameloblastoma, stereolithographic model, standardized formula

\section{INTRODUCTION}

Ameloblastoma is the most common benign odontogenic tumor of the jaw, and expansional growth of a huge untreated ameloblastoma can result in disturbances in facial aesthetics and function, such as difficulty with mouth opening, swallowing, chewing, breathing, neurologic deficits, and pathologic fractures. Radical wide resection with safety margins and subsequent reconstruction is generally recommended. ${ }^{1,2}$ A fibular free flap (FFF) is commonly used to reconstruct the mandible in order to adequately restore both aesthetic appearance and function. ${ }^{3}$

The aim of this communication report is to present a case of huge ameloblastoma after wide resection with free safety margins, and describe the immediate onestep mandibular reconstruction using a vascularized composite FFF.
The sterolithographic (rapid prototype, RP) model, a wax pattern of the resected mandible, and a surgical fibular stent made from the wax pattern were constructed preoperatively. Therefore, we suggest a standardized surgical protocol for mandibular reconstruction with FFF.

\section{CASE REPORT}

A 29-year-old female was referred to our department in 2009, with a chief complaint of pain during masticatory movement. Clinical examination and radiographic evaluation including computed tomography (CT) showed a huge mixed radiolucent and radiopaque lesion occupying the entire mandibular body and part of the ramus. Incisional biopsy via the intraoral mucosa confirmed as a follicular ameloblastoma. 


\section{Case Report}

This patient had a past medical history significant for schizophrenia and frequently missed her outpatient appointments. Additionally, she was homeless and had difficulty affording medical care. These circumstances made it difficult to provide appropriate evaluation and treatment of her condition. After hospitalization in a psychiatric institution, her schizophrenia was better controlled. With the help of a social welfare foundation, surgery for her growing mass was scheduled. For better treatment planning, a three-dimensional RP model was fabricated using CT data, and surgical margins were marked on the RP model. For the anticipated reconstruction using fibular bone, the base plate wax was duplicated using polysiloxane impression material. The stent did not exactly follow the outer surface of the mandible, but rather the simplest and most feasible design was obtained by twice bending the stent at the premolar area (Figure 1A). Preoperatively, angiography of the peroneal artery and its branches was performed to detect any vascular pathology or anatomical variations of the lower extremity, and cutaneous perforators were confirmed and marked with a Doppler flowmeter (Figure $1 \mathrm{~B}){ }^{4}$
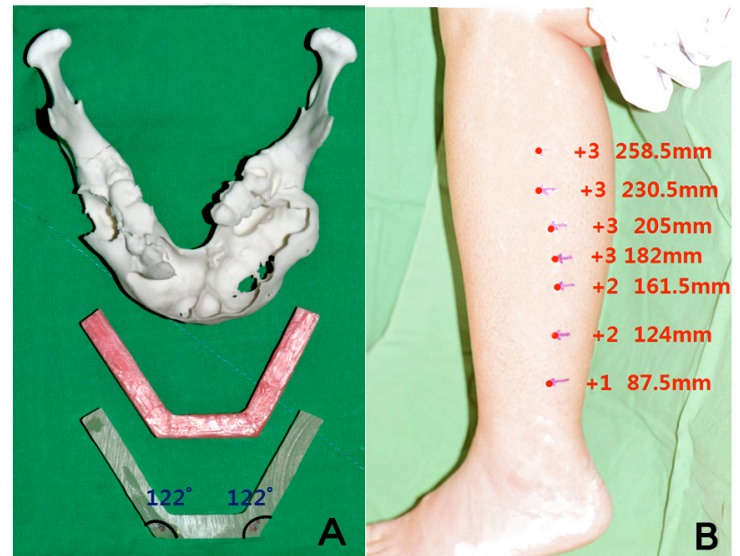

Figure 1 The 3-demensional RP model of the patient's mandible was compared with the wax and resin stent by twice bending at the premolar area (A). A lateral view of the patient's left lower leg with skin perforators showing the intensity of flow using a Doppler flowmeter (B).

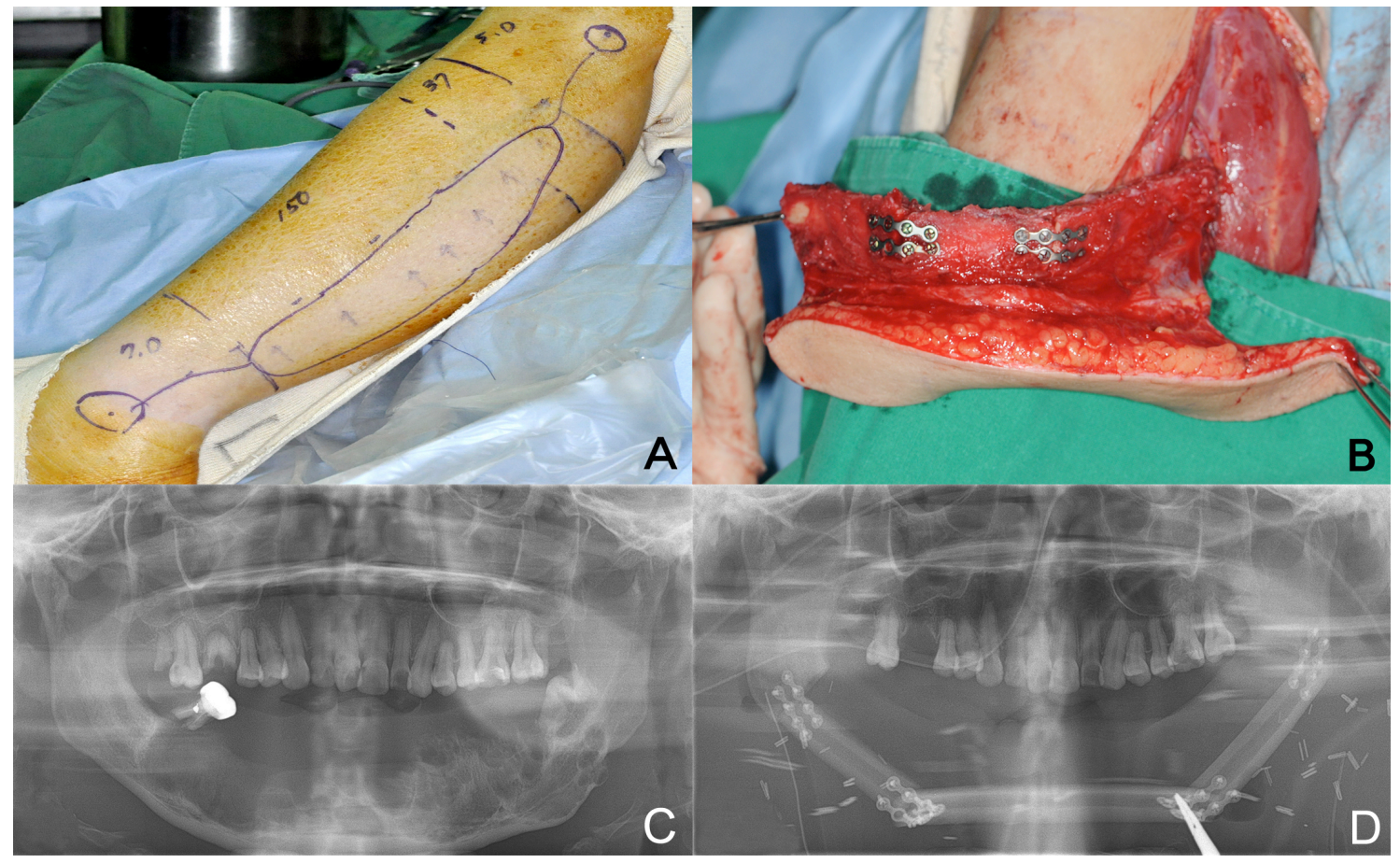

Figure 2 Preoperative skin marking according to the total fibular length with perforators in the distal third of the leg (A). The composite fibular free flap was harvested and bent according to the surgical template (B). Panoramic radiographs showing preoperative honeycomb-like osteolytic changes of the entire mandible with multiple cortical perforations $(\mathrm{C})$. View of the postoperative reconstructed mandible with minimal microplate fixation (D).

The neck incision line was made along the upper cervical crease from left to right at the mandibular angle. After blunt dissection, the left facial artery and internal jugular vein were identified and prepared for vessel anastomosis. Supraperiosteal mandibulectomy was performed and repositioning clamps were positioned on both resected margins to avoid displacement of the temporomandibular joints before detaching the mass. 
The $10.0 \times 4.0 \times 4.0 \mathrm{~cm}$ mass was resected en-block with most of the alveolar ridge and floating teeth included on both sides of the mandible. Microscopic examination showed proliferation of ameloblastic cells in a combination of several subtypes. Informed consent was obtained, and the Institutional Review Board of Seoul National University Dental Hospital approved the study.

After the skin paddle for the oral cavity mucosal reconstruction was outlined as shown in Figure 2A, the osteocutaneous FFF was harvested. After decuffing Toniquett and before detaching the peroneal vessels, the harvested fibula was contoured by wedge osteotomies using a reciprocating saw and rotating drill. According to the prefabricated surgical stent, the bent segment was fixated with 4-holes titanium miniplates ${ }^{\circledR}$ (KLS Martin Co., Germany) with a minimal vacant gap (Figure $2 \mathrm{~B}$ ). The composite FFF was transferred to the mandibular defect site under the guidance of the remaining condylar position with the repositioning clamp, and fixated with miniplates (Figures 2C and 2D). The skin island of the FFF was sufficient for the oral mucosal lining. End-toend vessel anastomosis of the peroneal artery to the facial artery and end-to-side anastomoses of two vena comitantes to the internal jugular vein were performed.

On postoperative Day 1, the tongue was held anteriorly by traction with tie suture to keep the airway clear and a Levin tube was inserted for feeding. The patient suffered from snoring when lying down, though this was reduced after one week and absent three weeks postoperatively. Physical therapy for movement of the lower facial musculature was encouraged. Oral feeding without a gag reflex was possible and pronunciation with facial expressions was natural after four weeks. After discharge, the patient experienced a favorable recovery without any donor or recipient site complications, and a favorable aesthetic and functional outcome was observed during the 28-month follow-up period.

\section{DISCUSSION}

Long-standing huge ameloblastoma can be extensive and extremely destructive, imposing a serious reduction in the quality of life of patients. In the past, mandibular defects presented a major challenge because of the lim- ited reconstruction options with a pedicled flap. However, current advances in microsurgery have allowed for composite soft and bony facial reconstruction. Fibula, scapula, and iliac crest flaps are commonly used as donor sites to reconstruct composite mandibular defects after ablative surgery. ${ }^{5}$

In conclusion, we preferred to use a vascularized osteocutaneous FFF for mandibular reconstruction based on several advantages: sufficient bone length, comparative ease of graft dissection and contouring, long pedicles with large caliber vessels, a two-team approach, minimal donor-site morbidity, and bicortical bone stock for dental implantation. Based on our experiences, we suggest that large untreated ameloblastomas that affect the entire mandibular body should be approached with radical treatment, and immediate reconstruction using FFF is a feasible option. A standardized protocol is suggested for the reconstruction of the entire mandibular body including pre- and intra-operative preparations.

\section{REFERENCES}

1. Bianchi B, Ferri A, Ferrari S, Leporati M, Copelli C, Ferri T, Sesenna E. Mandibular resection and reconstruction in the management of extensive amelobalstoma. J Oral Maxillofac Surg 2013;71:528537.

2. Becelli R, Morello R, Renzi G, Matarazzo G, Dominici C. Treatment of recurrent mandibular ameloblastoma with segmental resection and revascularized fibula free flap. J Craniofac Surg 2011;22:1163-1165.

3. Mijiti A, Ling W, Maimaiti A, Moming A. Singlestage management of huge desmoplastic ameloblastoma of the anterior mandible. J Plast Reconstr Aesthet Surg 2013;66:1440-1441.

4. Lee JH, Kim MJ, Kim JW. Mandibular reconstruction with free vascularized fibular flap. J Craniomaxillofac Surg 1995;23:20-26.

5. Zhu J, Yang Y, Li W. Assessment of quality of life and sociocultural aspects in patients with ameloblastoma after immediate mandibular reconstruction with a fibular free flap. Br J Oral Maxillofac Surg 2014;52:163-167. 